Pure and Applied Mathematics Quarterly

Volume 5, Number 2

(Special Issue: In honor of

Friedrich Hirzebruch, Part 1 of 2)

$853-872,2009$

\title{
Topological Modular Forms of Level 3
}

\author{
Mark Mahowald and Charles Rezk
}

\begin{abstract}
We describe and compute the homotopy of spectra of topological modular forms of level 3. We give some computations related to the "building complex" associated to level 3 structures at the prime 2. Finally, we note the existence of a number of connective models of the spectrum $\operatorname{TMF}\left(\Gamma_{0}(3)\right)$.
\end{abstract}

Keywords: elliptic cohomology, modular forms, topological modular forms, elliptic spectra, string bordism

\section{INTRODUCTION}

In this paper we collect a number of results related to the spectrum of "topological modular forms of level 3", denoted by $\operatorname{TMF}\left(\Gamma_{0}(3)\right)$. We investigated this spectrum as part of an attempt to describe the homotopy of the $K(2)$-local sphere (at the prime 2 ) in terms of modular forms. We think this work will be most useful in the context of a conjecture of Mark Behrens, which we describe below.

Along the way, we considered a cosimplicial spectrum associated to a certain "building", constructed using the moduli of certain kinds of subgroups of elliptic curves. The realization of this cosimplicial spectrum is denoted $Q(3)$; it is discussed in $\S 5$. Behrens conjectures [Beh, 1.6.1] that there is a cofiber sequence

$$
D_{K(2)} L_{K(2)} Q(3) \stackrel{D f}{\longrightarrow} \tilde{S} \stackrel{f}{\rightarrow} L_{K(2)} Q(3)
$$

Received October 10, 2006.

The second author was partially supported by National Science Foundation grants DMS-0203936 and DMS-0505056. 
where $D_{K(2)}$ denotes Spanier-Whitehead duality in the $K(2)$-local category (at the prime 2), and $\tilde{S}$ is a degree 2 Galois extension of the $K(2)$-local sphere. More precisely, $\tilde{S}=E_{2}^{h \tilde{\mathbb{G}}_{2}}$, where $\tilde{\mathbb{G}}_{2}=\tilde{\mathbb{S}}_{2} \rtimes$ Gal, with $\tilde{\mathbb{S}}_{2}$ an index 2 subgroup of the Morava stabilizer group, the kernel of

$$
\mathbb{S}_{2} \stackrel{N}{\longrightarrow} \mathbb{Z}_{2}^{\times} \rightarrow(\mathbb{Z} / 8)^{\times} /\{1,3\} .
$$

Behrens has proved a version of this conjecture at the prime 3 [Beh06].

In this paper we give results related to the calculation of the homotopy groups of $Q(3)$; we also describe how some known elements in the homotopy groups of spheres are detected in it. In doing so we give a complete calculation of the homotopy of $\operatorname{TMF}\left(\Gamma_{0}(3)\right)$. We also describe some connected versions of $\operatorname{TMF}\left(\Gamma_{0}(3)\right)$, which appear naturally in the cobordism spectrum $M$ String.

The spectrum $\operatorname{TMF}\left(\Gamma_{0}(3)\right)$, or one very much like it, has appeared in a different context. In [HK01], the authors construct "real" versions of the Johnson-Wilson spectra $E(n)$; these are $\mathbb{Z} / 2$-equivariant ring spectra, which admit an orientation by the "real" complex bordism spectrum. The homotopy fixed points of the $\mathbb{Z} / 2$-action on the $n$th real Johnson-Wilson spectrum is denoted $E R(n)$. As is clear from the work of $[\mathrm{KW}]$, the spectrum $E R(2)$ is very much like the spectrum $\operatorname{TMF}\left(\Gamma_{0}(3)\right)$. In particular, their calculation of $\pi_{*} E R(2)$ gives essentially the same answer as our calculation of $\pi_{*} \operatorname{TMF}\left(\Gamma_{0}(3)\right)$. (Note that $\operatorname{TMF}\left(\Gamma_{0}(3)\right)$ and $E R(2)$ are not identical as ring spectra, because they are $\mathbb{Z} / 2$-fixed points of $\operatorname{TMF}\left(\Gamma_{1}(3)\right)$ and $E(2)$ respectively, whose formal groups are not isomorphic. Presumably the construction of [HK01] can be carried out to construct a "real" version of $\operatorname{TMF}\left(\Gamma_{1}(3)\right)$.)

We would like to take this opportunity to dedicate this paper to Professor Hirzebruch; in particular, we would like to express our thanks for his book on modular forms [HBJ94].

\section{Spectra of topological MOdUlar forms}

In this section we describe the examples of spectra of topological modular forms we are interested in. It will be convenient to use the language of moduli stacks to identify them. Thus, let $\mathcal{M}$ denote the moduli stack of elliptic curves; an elliptic curve over a base scheme $S$ is a smooth proper morphism $E \rightarrow S$ whose geometric fibers are elliptic curves. Likewise, let $\overline{\mathcal{M}}$ denote the moduli stack of generalized elliptic curves; 
this is a compactification of $\mathcal{M}$ obtained by "adding the cusp". (See [KM85].) Thus, any morphism $f: S \rightarrow \overline{\mathcal{M}}$ determines a generalized elliptic curve $C_{f} \rightarrow S$.

There exists a line bundle $\omega \rightarrow \overline{\mathcal{M}}$, associated to the cotangent space of the identity section of a generalized elliptic curve. We write $\omega(C)$ for the line bundle over $S$ associated to the generalized elliptic curve $C \rightarrow S$. When $C$ is smooth, we can identify $\Gamma(S, \omega(C))$ with the set of invariant 1-forms on $C$. A modular form of level 1 and weight $k$ is a section of $\omega^{\otimes k} \rightarrow \overline{\mathcal{M}}$. Explicitly, a modular form of weight $k$ is a function $g$ which associates to each pair $(C \rightarrow S, \eta)$ with $C$ a generalized elliptic curve over $S$, and $\eta \in \Gamma(S, \omega)$, an element $g \in \mathcal{O}_{S}$, which is compatible with base change and such that $g(C, \lambda \eta)=\lambda^{-k} g(C, \eta)$ for $\lambda \in \mathcal{O}_{S}^{\times}$. We write $\operatorname{mf}_{k}=\Gamma\left(\overline{\mathcal{M}}, \omega^{\otimes k}\right)$.

An elliptic spectrum [AHS01] is a triple $(E, C, \phi)$ consisting of an even periodic ring spectrum $E$, a generalized elliptic curve $C$ over $\pi_{0} R$, and an isomorphism $\phi$ from the formal group of $E$ to the formal completion of $C$ at the identity. ("Even periodic" means: $\pi_{1} R=0$, and there exists $u \in \pi_{2} R$ which is invertible in the graded ring $\pi_{*} R$.)

All the examples of spectra of topological modular forms we need arise from the theorem of Goerss-Hopkins-Miller.

Theorem 2.1. There is a sheaf $\mathcal{O}_{\text {tmf }}$ of $E_{\infty}$-ring spectra over the stack $\overline{\mathcal{M}}$ in the étale topology, which has the following property. For étale morphisms $f: \operatorname{Spec}(R) \rightarrow \overline{\mathcal{M}}$, there is a natural structure of elliptic spectrum $\left(\mathcal{O}_{\operatorname{tmf}}(f), C_{f}, \phi\right)$, where $\pi_{0} \mathcal{O}_{\text {tmf }}(f)=R$ and $C_{f}$ is the generalized elliptic curve over $R$ classified by $f$.

As a consequence of this result, there is a spectral sequence

$$
E_{2}=H^{s}\left(\mathcal{N}, f^{*} \omega^{\otimes k}\right) \Longrightarrow \pi_{2 k-s} \mathcal{O}_{\mathrm{tmf}}(f)
$$

associated to any étale map $f: \mathcal{N} \rightarrow \overline{\mathcal{M}}$. The spectral sequence is functorial in $\mathcal{N}$. If $\mathcal{N}=\operatorname{Spec}(R)$ is an affine scheme, the spectral sequence collapses at $E_{2}$ and we have $\pi_{2 k} \mathcal{O}_{\mathrm{tmf}}(f)=\Gamma\left(\operatorname{Spec}(R), f^{*} \omega^{\otimes k}\right)$.

Here are some of the basic examples we care about.

(1) Let tmf denote the $(-1)$-connected cover of $\mathcal{O}_{\text {tmf }}($ id: $\overline{\mathcal{M}} \rightarrow \overline{\mathcal{M}})$. This is the connective spectrum of topological modular forms, first constructed by Hopkins, Miller, and the first author; some details are given in [HM].

(2) Let $\mathrm{TMF}=\mathcal{O}_{\mathrm{tmf}}(\mathcal{M} \rightarrow \overline{\mathcal{M}})$, the periodic spectrum of topological modular forms; the periodic invertible element is $\Delta^{24} \in \pi_{576} \mathrm{TMF}$. 
Another family of examples comes from introducing a level 3 structure. Let $S$ be a scheme over $\mathbb{Z}\left[\frac{1}{3}\right]$. If $C \rightarrow S$ is an elliptic curve, let $C[3]$ denote the scheme of "3torsion points" of $C$ (that is, the kernel of [3]: $C \rightarrow C$ ). Then $C[3] \rightarrow S$ is an finite étale morphism (of degree 9). Locally in the étale topology on $S, C[3]$ is isomorphic to the discrete group scheme $\mathbb{Z} / 3 \times \mathbb{Z} / 3$.

(1) A $\Gamma(3)$ structure on $C \rightarrow S$ is a choice of isomorphism $\mathbb{Z} / 3 \times \mathbb{Z} / 3 \rightarrow C[3]$ of group schemes over $S$. Equivalently, a $\Gamma(3)$-structure is a choice of a pair of sections $s_{1}, s_{2}$ of $C[3] \rightarrow S$ which are (locally in $S$ ) linearly independent.

(2) A $\Gamma_{1}(3)$ structure on $C \rightarrow S$ is a choice of monomorphism $\mathbb{Z} / 3 \rightarrow C[3]$ of group schemes over $S$. Equivalently, a $\Gamma_{1}(3)$-structure is a choice of a (locally non-identity) section of $C[3] \rightarrow S$.

(3) $\mathrm{A} \Gamma_{0}(3)$ structure on $C \rightarrow S$ is a choice of subgroup scheme $A \subseteq C[3]$ over $S$ which is isomorphic to $\mathbb{Z} / 3$. Equivalently, a $\Gamma_{0}(3)$-structure is a choice of equivalence class of $\Gamma_{1}(3)$-structures, where we identify sections which are carried to one another by the inversion $[-1]: C[3] \rightarrow C[3]$.

Each of these notions of level structure has an associated moduli stack, and there are finite étale morphisms

$$
\mathcal{M}(\Gamma(3)) \rightarrow \mathcal{M}\left(\Gamma_{1}(3)\right) \rightarrow \mathcal{M}\left(\Gamma_{0}(3)\right) \rightarrow \mathcal{M}\left[\frac{1}{3}\right]
$$

of degrees 6, 2, and 4 respectively. Associated to these are maps of $E_{\infty}$-rings $\operatorname{TMF}\left[\frac{1}{3}\right] \rightarrow \operatorname{TMF}\left(\Gamma_{0}(3)\right) \rightarrow \operatorname{TMF}\left(\Gamma_{1}(3)\right) \rightarrow \operatorname{TMF}(\Gamma(3))$. Thus we obtain a spectrum of topological modular forms $\operatorname{TMF}(\Gamma(3))$, and similarly for $\Gamma(3)$ replaced with $\Gamma_{1}(3)$ and $\Gamma_{0}(3)$. All these spectra are $E_{\infty}$-rings under the commutative ring spectrum $S\left[\frac{1}{3}\right]$.

Remark 2.2. These notions of level structure admit generalizations to generalized elliptic curves over arbitrary schemes $S$ (i.e., without requiring 3 to be inverted, and allowing non-smooth curves). However, the resulting moduli stacks are not étale over $\overline{\mathcal{M}}$, and they won't play a role in this paper. See [KM85].

Let $G=G L(2, \mathbb{Z} / 3)$. It is clear that this group acts on the set of $\Gamma(3)$-structures of an elliptic curve, and thus acts on $\operatorname{TMF}(\Gamma(3))$. In particular, it is the Galois group of the finite étale cover $\mathcal{M}(\Gamma(3)) \rightarrow \mathcal{M}$, and thus we have that $\operatorname{TMF}\left[\frac{1}{3}\right] \approx \operatorname{TMF}(\Gamma(3))^{h G}$. Let

$$
G_{\Gamma_{1}(3)}=\left\{\left(\begin{array}{c}
1 * \\
0 *
\end{array}\right) \in G\right\} \approx \mathbb{Z} / 3 \ltimes \mathbb{Z} / 2
$$


and

$$
G_{\Gamma_{0}(3)}=\left\{\left(\begin{array}{c}
* * \\
0 *
\end{array}\right) \in G\right\} \approx \mathbb{Z} / 6 \ltimes \mathbb{Z} / 2 .
$$

Then we have $\operatorname{TMF}\left(\Gamma_{1}(3)\right) \approx \operatorname{TMF}(\Gamma(3))^{h G_{\Gamma_{1}(3)}}$ and $\operatorname{TMF}\left(\Gamma_{0}(3)\right) \approx \operatorname{TMF}(\Gamma(3))^{h G_{\Gamma_{0}(3)}}$. Furthermore, since $G_{\Gamma_{1}(3)}$ is normal of index 2 in $G_{\Gamma_{0}(3)}$, we have $\operatorname{TMF}\left(\Gamma_{0}(3)\right) \approx$ $\operatorname{TMF}\left(\Gamma_{1}(3)\right)^{h \mathbb{Z} / 2}$.

A Weierstrass curve over a ring $R$ is the closure $C_{\underline{a}}=C_{\left(a_{1}, \ldots, a_{6}\right)}$ in $\mathbb{P}_{R}^{2}$ of an affine curve of the form

$$
y^{2}+a_{1} x y+a_{3} y=x^{3}+a_{2} x^{2}+a_{4} x+a_{6},
$$

with $a_{1}, \ldots, a_{6} \in R$. A Weierstrass curve is smooth if and only if the discriminant $\Delta=\Delta\left(a_{1}, \ldots, a_{6}\right)$ is invertible in $R$. An isomorphism $C_{\underline{a}^{\prime}} \rightarrow C_{\underline{a}}$ between Weierstrass curves is an algebraic map given by

$$
x \mapsto \lambda^{-2} x+r, \quad y \mapsto \lambda^{-3} y+\lambda^{-1} s x+t
$$

which sends $C_{\underline{a}^{\prime}}$ into $C_{\underline{a}}$. There is an Artin stack $\mathcal{M}_{\text {Weier }}$ of Weierstrass curves, determined by the Hopf algebroid implicitly described above.

Every generalized elliptic curve $C \rightarrow S$ admits a presentation, locally over $S$ in the flat topology, as a Weierstrass curve, in which the identity element of the elliptic curve corresponds to the unique point at infinity on the Weierstrass curve. Thus there is an open embedding $\overline{\mathcal{M}} \rightarrow \mathcal{M}_{\text {Weier }}$. There is a line bundle $\omega$ over $\mathcal{M}_{\text {Weier }}$ generated by invariant differential

$$
\eta_{\underline{a}}=\frac{d x}{2 y+a_{1} x+a_{3}}=\frac{d y}{3 x^{2}+2 a_{2} x+a_{4}-a_{1} y},
$$

and this pulls back to the line bundle $\omega$ over $\overline{\mathcal{M}}$.

In particular, this means that the ring $\mathrm{mf}_{*}$ of level 1-modular is a subring of $A=\mathbb{Z}\left[a_{1}, a_{2}, a_{3}, a_{4}, a_{6}\right]$, and there are polynomials $C_{4}, C_{6} \in A$ such that for any Weierstrass curve $C_{\underline{a}}, c_{i}\left(C_{\underline{a}}, \eta\right)=C\left(a_{1}, \ldots, a_{6}\right)$. These polynomials are those of [Del75], [Sil86, p. 46].

\section{Modular fORMS OF LEVEL 3}

Explicit calculations about level 3 structures flow from the following observation. 
Proposition 3.1. Let $C=C_{\underline{a}} \subset \mathbb{P}^{2}$ be a smooth Weierstrass curve over a ring $R$ in which 3 is invertible. An $R$ point $P$ on $C$ has order 3 if and only if it is a flex point; that is, if and only if the tangent line $L$ at $P$ makes a triple intersection with $C$ at $P$.

Proof. This is a consequence of the description of the group structure on a smooth Weierstrass curve. If $L \subset \mathbb{P}^{2}$ is a line and $P_{1}, P_{2}, P_{3}$ its three points of intersection with $C$, counted with multiplicity, then $\left[P_{1}\right]+\left[P_{2}\right]+\left[P_{3}\right]=0$ in the group structure of $C$. (See [Sil86] or [KM85].)

We give a concrete description of $\mathcal{M}\left(\Gamma_{1}(3)\right)$.

Proposition 3.2. Let $R$ be in which 3 is invertible. Let $(C, \eta, P)$ be a smooth elliptic curve $C$ over $R$ together with an invariant 1-form $\eta$ on $C$ and a $\Gamma_{1}(3)$-structure $P \in C(R)$. Then there is a unique triple $\left(C_{1}, \eta_{1}, P_{1}\right)$ and a unique isomorphism $\left(C_{1}, \eta_{1}, P_{1}\right) \approx(C, \eta, P)$ of this data such that

$$
C_{1}: \quad y^{2}+A_{1} x y+A_{3} y=x^{3}, \quad \eta_{1}=\frac{d x}{2 y+A_{1} x+A_{3}}=\frac{d y}{3 x^{2}-A_{1} y}
$$

with $\Delta=A_{3}^{3}\left(A_{1}^{3}-27 A_{3}\right)$ invertible in $R$, and such that $P_{1}$ is the point with $x y$ coordinates $(0,0)$.

In particular, the moduli problem of triples $(C, \eta, P)$ as above is represented by the affine scheme $\mathbb{Z}\left[\frac{1}{3}, A_{1}, A_{3}, \Delta^{-1}\right]$.

Proof. Note that on the Weierstrass curve $C_{1}$, the tangent line to $P_{1}$ is precisely the $x$-axis (so that $P_{1}$ is indeed a point of order 3 by (3.1)). The result amounts to showing that given $(C, \eta, P)$ where $C$ is a smooth Weierstrass curve, there is a unique Weierstrass transformation sending $P$ to the origin and sending the tangent line at $P$ to the $x$-axis. This is easiest to see in three steps. Given a pair $(C, P)$ where $C$ is a Weierstrass curve and $P=(\alpha, \beta)$ a point of exact order 3 , use the variable substitution $x^{\prime}=x+\alpha, y^{\prime}=y+\beta$ to get a Weierstrass curve $C^{\prime}$ of the form

$$
y^{2}+A_{1} x y+A_{3} y=x^{3}+A_{2} x^{2}+A_{4} x,
$$

so that $P \in C$ corresponds with $P^{\prime}=(0,0) \in C^{\prime}$. The tangent line $L$ at $P^{\prime}$ is given by $A_{3} y=A_{4} x$, and since $L$ cannot be vertical (every vertical line in the $x y$ plane intersects the curve at the infinity), we have that $A_{3}$ is invertible. Thus the transformation $x^{\prime \prime}=x^{\prime}, y^{\prime \prime}=y^{\prime}+\left(A_{4} / A_{3}\right) x^{\prime}$ gives rise to a new Weierstrass curve $C^{\prime \prime}$, which must have the desired form, since $P^{\prime \prime}=(0,0)$ must be a triple intersection point 
of $C^{\prime \prime}$ with the $x$-axis. Finally use a transformation of the form $x^{\prime \prime \prime}=\lambda^{-2} x^{\prime \prime}, y^{\prime \prime \prime}=$ $\lambda^{-3} y^{\prime \prime}$ to get the invariant differential in the right form.

Corollary 3.3. We have

$$
\pi_{*} \operatorname{TMF}\left(\Gamma_{1}(3)\right) \approx H^{0}\left(\mathcal{M}, \omega^{\otimes *}\right) \approx \mathbb{Z}\left[\frac{1}{3}, a_{1}, a_{3}, \Delta^{-1}\right],
$$

where $\left|a_{1}\right|=2$ and $\left|a_{3}\right|=6$.

Proof. By $(3.2), H^{*}\left(\mathcal{M}\left(\Gamma_{1}(3)\right), \omega^{\otimes *}\right)$ is equal to the cohomology of the Hopf algebroid with $A=\mathbb{Z}\left[\frac{1}{3}, a_{1}, a_{3}, \Delta^{-1}\right]$ and $\Gamma=A\left[\lambda, \lambda^{-1}\right]$.

Note that for a curve of the form $y^{2}+a_{1} x y+a_{3} y=x^{3}$, the inversion map is given by $[-1](x, y)=\left(x,-y-a_{1} x-a_{3}\right)$. In particular, the point $-P$ has $x y$-coordinates $\left(0,-a_{3}\right)$.

Proposition 3.4. We have $H^{s}\left(\mathcal{M}\left(\Gamma_{0}(3)\right), \omega^{\otimes k}\right) \approx H^{s}\left(\mathbb{Z} / 2, \mathbb{Z}\left[\frac{1}{3}, a_{1}, a_{3}, \Delta^{-1}\right]\right)$, where the generator of $\sigma$ of $\mathbb{Z} / 2$ acts by $\sigma\left(a_{1}\right)=-a_{1}$ and $\sigma\left(a_{3}\right)=-a_{3}$. In particular, there is a spectral sequence $E_{r}\left(\operatorname{TMF}\left(\Gamma_{0}(3)\right)\right)$, with

$$
E_{2}^{s, t}\left(\operatorname{TMF}\left(\Gamma_{0}(3)\right)\right) \approx H^{s}\left(\mathbb{Z} / 2, \pi_{t} \operatorname{TMF}\left(\Gamma_{1}(3)\right)\right) \Longrightarrow \pi_{t-s} \operatorname{TMF}\left(\Gamma_{0}(3)\right) .
$$

Recall that the spectral sequence of (3.4) is that computing homotopy groups of the homotopy fixed point spectrum $\operatorname{TMF}(\Gamma(3))^{h G_{\Gamma_{0}(3)}}$. Thus there is a comparison map $E_{r}(\mathrm{TMF}) \rightarrow E_{r}\left(\mathrm{TMF}\left(\Gamma_{0}(3)\right)\right)$ with the spectral sequence for $\mathrm{TMF}=\mathrm{TMF}^{h G}$. Associated to the natural map of commutative ring spectra $f^{*}: \operatorname{TMF} \rightarrow \operatorname{TMF}\left(\Gamma_{0}(3)\right)$ there is a transfer map $f_{!}: \operatorname{TMF}\left(\Gamma_{0}(3)\right) \rightarrow$ TMF, which is a map of TMF-module spectra. We note that there is a map of spectral sequences $f_{!}: E_{r}\left(\operatorname{TMF}\left(\Gamma_{0}(3)\right)\right) \rightarrow$ $E_{r}(\mathrm{TMF})$, and that on $E_{2}$-terms, this map is precisely the cohomology transfer. In particular, we have

Proposition 3.5. The composite $\mathrm{TMF} \stackrel{f^{*}}{\longrightarrow} \mathrm{TMF}\left(\Gamma_{0}(3)\right) \stackrel{f_{!}}{\longrightarrow} \mathrm{TMF}$ is equal to $4 \mathrm{id}$.

Proof. Since both are maps of TMF modules, we only have to check the image of $1 \in \pi_{0} \mathrm{TMF}$. It is a straightforward fact about group cohomology that the composite $H^{*}\left(G, \pi_{*} \operatorname{TMF}(\Gamma(3)) \rightarrow H^{*}\left(G_{\Gamma_{0}(3)}, \pi_{*} \operatorname{TMF}(\Gamma(3))\right) \rightarrow H^{*}\left(G, \pi_{*} \operatorname{TMF}(\Gamma(3))\right)\right.$ is given by multiplication by 4 . The result follows from the fact that $E_{\infty}^{s, s}(\mathrm{TMF})=0$ for $s>0$. 


\section{TOPOlOGical MOdUlar FORMS OF LEVEL 3}

The $E_{2}$-term can be described in terms of the bigraded ring $R^{s, t}=\mathbb{Z}\left[\frac{1}{3}, a_{1}, a_{3}, \zeta, \Delta^{-1}\right]$ $/(2 \zeta)$, where $\zeta$ is given bidegree $(1,0)$. If we assign "odd" weight to $a_{1}, a_{3}$, and $\zeta$, then $E_{2}=H^{s}\left(\mathbb{Z} / 2, \pi_{t} \operatorname{TMF}\left(\Gamma_{1}(3)\right)\right)$ can be identified with the even part of $R^{s, t}$.

Let $x=\zeta a_{3}^{3} \in E_{2}^{1,18}$. There is an isomorphism

$$
E_{2} \approx \mathbb{Z}\left[\frac{1}{3}, a_{1}^{2}, a_{1} a_{3}, a_{3}^{2}, \Delta^{-1}\right][x] /(2 x) .
$$

We write

$$
h_{1}=\zeta a_{1}=x a_{1} a_{3} a_{3}^{-4}, \quad h_{2}=\zeta^{3} a_{3}=x^{3} a_{3}^{-8}, \quad h_{2,0}=\zeta a_{3}=x a_{3}^{-2} .
$$

We have the following $d_{3}$ differentials:

$$
d_{3}: a_{1}^{2} \mapsto h_{1}^{3}, \quad a_{3}^{2} \mapsto h_{1} h_{2,0}^{2}, \quad a_{1} a_{3} \mapsto 0, \quad h_{1} \mapsto 0 .
$$

In general, if $c \in R^{0, t}$ has odd weight, then $d_{3}\left(c^{2}\right)=h_{1}(\zeta c)^{2}$; this can be proved by a cup-1 construction. We can identify $h_{1}$ as the class representing the image of $\eta \in \pi_{1} S^{0}$. We will show in (6.2) that $a_{1} a_{3}$ is a permanent cycle.

This forces:

$$
d_{3}: h_{2,0} \mapsto h_{1} h_{2,0} \zeta^{2}, \quad x \mapsto 0 .
$$

(The differentials already computed show that $d_{3}: E_{3}^{4,20} \rightarrow E_{3}^{7,22}$ is injective, so $d_{3}(x)=0$, and $h_{2,0}=x a_{3}^{-2}$.)

We note that the transfer argument of (3.5) shows that whenever there is an element $\alpha$ of order 8 in $\pi_{*} \mathrm{TMF}$, its image $\alpha^{\prime}$ in $\pi_{*} \operatorname{TMF}\left(\Gamma_{0}(3)\right)$ is non-trivial. Furthermore, if $\alpha$ and $4 \alpha$ are detected on the $s_{0}$-line and $s_{1}$-line of $E_{\infty}(\mathrm{TMF})$, respectively, then $\alpha^{\prime}$ must be detected on the $s$-line of $E_{\infty}\left(\operatorname{TMF}\left(\Gamma_{0}(3)\right)\right)$, where $s_{0} \leq s \leq s_{1}$. This allows us to see that

$$
h_{2}=\zeta^{3} a_{3} \in E_{2}^{3,6} \quad \text { detects } \quad \nu \in \pi_{3} \operatorname{TMF}\left(\Gamma_{0}(3)\right),
$$

and

$$
h_{2,0}^{4}=\zeta^{4} a_{3}^{4} \in E_{2}^{4,24} \quad \text { detects } \quad \bar{\kappa} \in \pi_{20} \operatorname{TMF}\left(\Gamma_{0}(3)\right),
$$

where these elements are images of the like-named classes in $\pi_{*} S^{0}$.

At this point there are no possible differentials until the $E_{7}$-term. There is a map $E_{7} \rightarrow \mathbb{F}_{2}\left[\Delta, \Delta^{-1}, x\right]$ which is surjective, and is an isomorphism on lines $s \geq 3$.

The element $h_{2,0}^{4}=x^{4} \Delta^{-1}$ is the image of the class representing $\bar{\kappa}$ in the spectral sequence for $\pi_{*}$ TMF, so it is a permanent cycle. There is a relation $\bar{\kappa}^{6}=0$ in $\pi_{*}$ TMF, 
and hence this relation must hold in $\pi_{*} \operatorname{TMF}\left(\Gamma_{0}(3)\right)$. The only possible differential which can do this is is $d_{7}\left(x^{17} \Delta^{-7}\right)=\left(h_{2,0}^{4}\right)^{6}$.

This implies that $d_{7}$ is non-trivial on either $x$ or on $\Delta^{4}$ (but not both). In either case, one sees that $E_{7}=E_{\infty}$, and that this vanishes for lines $s \geq 7$.

Thus, we see that $x$ is a permanent cycle, whence $d_{7}(\Delta)=h_{2,0}^{4} \nu$.

Proposition 4.1. The above provides a description of $\pi_{*} \operatorname{TMF}\left(\Gamma_{0}(3)\right)$. In particular, there is an exact sequence

$$
\begin{aligned}
0 \rightarrow \mathbb{F}_{2}\left[\Delta^{ \pm 2}\right]\left\{\nu, \nu^{2}, x, \eta x, \bar{\kappa}, x^{2}, \nu x^{2}\right\} \rightarrow \pi_{*} \operatorname{TMF}\left(\Gamma_{0}(3)\right) & \\
& \rightarrow \operatorname{bo}_{*}\left[\frac{1}{3}, \Delta^{ \pm 1}\right]\left\{1, a_{1} a_{3}\right\} \oplus \operatorname{bsp}_{*}\left[\frac{1}{3}, \Delta^{ \pm 1}\right]\left\{2 a_{3}^{2}, 2\left(a_{1} a_{3}\right) a_{3}^{2}\right\} \rightarrow \Delta \mathbb{F}_{2}\left[\Delta^{ \pm 2}\right] \rightarrow 0 .
\end{aligned}
$$

The spectrum $\operatorname{TMF}\left(\Gamma_{0}(3)\right)$ is 48-periodic, with periodicity generated by $\Delta^{2}$. The elements $\eta, \nu$, and $\bar{\kappa}$ are images of the like-named elements in the 1, 3 and 20 stems of $S^{0}$. The element $x$ lies in $\pi_{17} \operatorname{TMF}\left(\Gamma_{0}(3)\right)$. Furthermore, this sequence encodes the multiplicative structure, except that in addition we must note that $a_{1}^{4} x=\eta\left(a_{1} a_{3}\right)^{3}$, $a_{1} a_{3} x=\eta \Delta, a_{1} a_{3} x^{2}=\eta^{2}\left(a_{1} a_{3}\right) a_{3}^{4}, \nu x=\bar{\kappa}$, and that $x^{3}=\nu \Delta^{2}, x^{4}=\bar{\kappa} \Delta^{2}$, $x^{5}=\nu x \Delta^{2}, x^{6}=\nu^{2} \Delta^{4}$, and $x^{7}=0$.

Remark 4.2. It is notable how element $x$ generates all the $v_{1}$-periodic torsion in $\pi_{*} \operatorname{TMF}\left(\Gamma_{0}(3)\right)$. It is particularly nice since $x$ and $x^{2}$ are $v_{1}^{4}$ periodic. An elementary calculation on homotopy groups shows that there is a cofiber sequence

$$
\Sigma^{17} \operatorname{TMF}\left(\Gamma_{0}(3)\right) \stackrel{x \cdot}{\rightarrow} \operatorname{TMF}\left(\Gamma_{0}(3)\right) \rightarrow \operatorname{TMF}\left(\Gamma_{1}(3)\right)
$$

in the category of $\operatorname{TMF}\left(\Gamma_{0}(3)\right)$-module spectra. This seems to be an example of one of the fibrations produced in $[\mathrm{KW}]$, where such fibrations are constructed for all the "real" Johnson-Wilson spectra.

\section{Hecke operators, ANd the Building Complex}

In this section we describe a version of the "building complex" which has been studied fruitfully by Behrens [Beh06]. In the following, we assume that all schemes $S$ are defined over $\mathbb{Z}\left[\frac{1}{3}\right]$.

We define a semi-simplicial stack $\mathcal{N}_{\bullet}$, together with a "line bundle" $\omega_{\bullet}$ over $\mathcal{N}_{\bullet}$, as follows. Let $\mathcal{N}_{k}$ be the moduli stack of data of the form

$$
C_{0} \stackrel{\phi_{1}}{\longrightarrow} C_{1} \stackrel{\phi_{2}}{\longrightarrow} \cdots \stackrel{\phi_{k}}{\longrightarrow} C_{k}
$$


where the $C_{i}$ are smooth elliptic curves over a base $S$, and the $\phi_{i}$ are isogenies of elliptic curves which are not isomorphisms, such that $\operatorname{ker}\left(\phi_{k} \cdots \phi_{1}\right) \subseteq C_{0}[3]$. In particular, each $\phi_{i}$ has degree either 3 or $3^{2}$. Face maps are defined in the evident way. It is readily apparent that $\mathcal{N}_{k}$ is empty for $k>2$.

The semi-simplicial stack can be completed to a simplicial stack by formally adding degeneracies (which amounts to allowing some of the $\phi_{i}$ to be isomorphisms).

To give the line bundle $\omega_{\bullet}$, we set $\omega_{k}$ to be the line bundle over $\mathcal{N}_{k}$ defined by $\omega_{k}=\omega\left(C_{0}\right)$, together with "descent data" specified by the following isomorphisms

$$
\begin{array}{ll}
\left(d_{j}^{*} \omega_{k-1} \stackrel{\sim}{\longrightarrow} \omega_{k}\right)=\left(\text { id }: \omega\left(C_{0}\right) \rightarrow \omega\left(C_{0}\right)\right) & \text { for } 0<j \leq k, \\
\left(d_{0}^{*} \omega_{k-1} \stackrel{\sim}{\longrightarrow} \omega_{k}\right)=\left(\phi_{1}^{*}: \omega\left(C_{1}\right) \rightarrow \omega\left(C_{0}\right)\right), &
\end{array}
$$

where $\phi_{1}^{*}: \omega\left(C_{1}\right) \rightarrow \omega\left(C_{0}\right)$ is the map induced by pulling back 1-forms; since 3 is inverted in the ground ring, these are isomorphisms.

We now describe all the structure here in terms of more familiar objects, making use of the fact that the moduli of isogenies $C_{0} \rightarrow C_{1}$ is equivalent to the moduli of pairs $\left(C_{0}, A\right)$, consisting of a curve $C_{0}$ and a finite subgroup $A$. Thus, consider the following morphisms of moduli stacks.

$$
\begin{array}{lll}
h: \mathcal{M} \rightarrow \mathcal{M}, & & (C) \mapsto(C / C[3]), \\
f: \mathcal{M}\left(\Gamma_{0}(3)\right) \rightarrow \mathcal{M}, & & (A<C) \mapsto(C), \\
q: \mathcal{M}\left(\Gamma_{0}(3)\right) \rightarrow \mathcal{M}, & & (A<C) \mapsto(C / A), \\
t: \mathcal{M}\left(\Gamma_{0}(3)\right) \rightarrow \mathcal{M}\left(\Gamma_{0}(3)\right), & & (A<C) \mapsto(C[3] / A<C / A) .
\end{array}
$$

We have described the morphisms in terms of the effect on objects. For instance, $q: \mathcal{M}\left(\Gamma_{0}(3)\right) \rightarrow \mathcal{M}$ is the morphism of stacks associated to the operation which sends the data of a smooth elliptic curve $C$, together with a subgroup scheme $A<C$ locally isomorphic to $\mathbb{Z} / 3$, and produces the quotient group scheme $C / A$, which is again an elliptic curve. The notation $C[3]$ denotes the subgroup of 3-torsion points in $C$; under our hypotheses on the base scheme, $C[3]$ is an etale group scheme locally isomorphic to $\mathbb{Z} / 3 \times \mathbb{Z} / 3$. 
We may now describe the semi-simplicial stack $\mathcal{N}_{\bullet}$ by the following picture.

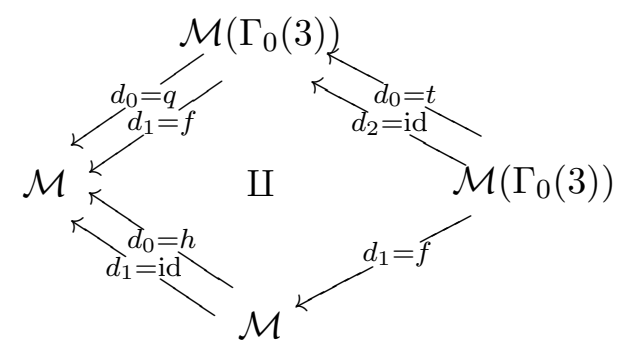

$$
\mathcal{N}_{0} \quad \mathcal{N}_{1} \quad \mathcal{N}_{2}
$$

The simplicial identities follow from the identities

$$
q=f t \quad \text { and } \quad q t=h f .
$$

The line bundle $\omega_{\bullet}$ is described by

$$
\omega_{0}=\omega,\left.\quad \omega_{1}\right|_{\mathcal{M}\left(\Gamma_{0}(3)\right)}=f^{*} \omega,\left.\quad \omega_{1}\right|_{\mathcal{M}}=\omega, \quad \omega_{2}=f^{*} \omega,
$$

with the non-trivial parts of the descent data given by the maps $q^{*} \omega \stackrel{\sim}{\longrightarrow} f^{*} \omega$ and $h^{*} \omega \stackrel{\sim}{\longrightarrow} \omega$ induced by isogenies.

There is an associated semi-cosimplicial commutative ring spectrum

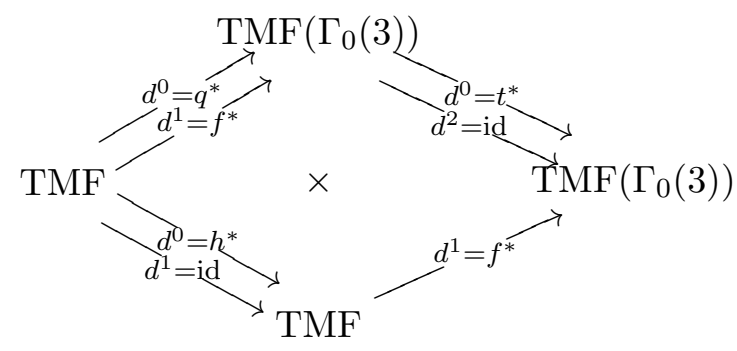

Following Behrens, we write $Q(3)$ for the geometric realization of the cosimplicial ring

There is also a cosimplicial bigraded ring given by $H^{*}\left(\mathcal{N}_{\bullet}, \omega^{\otimes *}\right)$; we also write $f^{*}, q^{*}, t^{*}, h^{*}$ for the induced maps on cohomology. In this section we compute the effect of these maps. 
Write $\operatorname{MF}_{k}=H^{0}\left(\mathcal{M}, \omega^{\otimes k}\right)$ and $\operatorname{MF}\left(\Gamma_{0}(3)\right)_{k}=H^{0}\left(\mathcal{M}\left(\Gamma_{0}(3)\right), \omega^{\otimes k}\right)$ for the rings of modular forms. Recall that

$$
\begin{aligned}
\mathrm{MF}_{*} & =\mathbb{Z}\left[c_{4}, c_{6}, \Delta, \Delta^{-1}\right] /\left(c_{4}^{3}-c_{6}^{2}-1728 \Delta\right), \\
\operatorname{MF}\left(\Gamma_{0}(3)\right)_{*} & =\mathbb{Z}\left[\frac{1}{3}, a_{1}^{2}, a_{3}^{2}, a_{1} a_{3},\left(a_{1}^{3} a_{3}^{3}-27 a_{3}^{4}\right)^{-1}\right] .
\end{aligned}
$$

Proposition 5.2. The maps $f^{*}: \mathrm{MF} \rightarrow \mathrm{MF}\left(\Gamma_{0}(3)\right), h^{*}: \mathrm{MF} \rightarrow \mathrm{MF}, q^{*}: \mathrm{MF} \rightarrow$ $\operatorname{MF}\left(\Gamma_{0}(3)\right)$, and $t^{*}: \operatorname{MF}\left(\Gamma_{0}(3)\right) \rightarrow \operatorname{MF}\left(\Gamma_{0}(3)\right)$, are described by

$$
\begin{array}{cc}
f^{*}\left(c_{4}\right)=a_{1}^{4}-24 a_{1} a_{3}, & h^{*}\left(c_{4}\right)=3^{4} c_{4}, \\
f^{*}\left(c_{6}\right)=-a_{1}^{6}+36 a_{1}^{3} a_{3}-216 a_{3}^{2}, & h^{*}\left(c_{6}\right)=3^{6} c_{6}, \\
f^{*}(\Delta)=a_{1}^{3} a_{3}^{3}-27 a_{3}^{4}, & h^{*}(\Delta)=3^{12} \Delta . \\
q^{*}\left(c_{4}\right)=a_{1}^{4}+216 a_{1} a_{3}, & \\
q^{*}\left(c_{6}\right)=-a_{1}^{6}+540 a_{1}^{3} a_{3}+5832 a_{3}^{2}, & \\
q^{*}(\Delta)=a_{1}^{9} a_{3}-81 a_{1}^{6} a_{3}^{2}+2187 a_{1}^{3} a_{3}^{3}-19683 a_{3}^{4} . \\
t^{*}\left(a_{1}^{2}\right)=-3 a_{1}^{2}, \\
t^{*}\left(a_{1} a_{3}\right)=\frac{1}{3} a_{1}^{4}-9 a_{1} a_{3}, \\
t^{*}\left(a_{3}^{2}\right)=-\frac{1}{27} a_{1}^{6}+2 a_{1}^{3} a_{3}-27 a_{3}^{2} .
\end{array}
$$

We will give the proof of (5.2) at the end of the section.

Let $A=\mathbb{Z}\left[\frac{1}{3}, a_{1}, a_{3}, \Delta^{-1}\right]$; let $C$ denote the curve given by Weierstrass equation $y^{2}+a_{1} x y+a_{3} y=x^{3}$, and let $\eta=\frac{d x}{2 y+a_{1} x+a_{3}}$ denote the usual invariant 1-form. Thus $C$ is a model for the universal curve over $\mathcal{M}\left(\Gamma_{1}(3)\right)$, with $P_{0}=(0,0)$ as the distinguished point of order 3 , and $-P_{0}=\left(0,-a_{3}\right)$.

Proposition 5.3. Let $C^{\prime}$ denote the Weierstrass curve over $A$ defined by the affine equation

$$
Y^{2}+a_{1} X Y+3 a_{3} Y=X^{3}-6 a_{1} a_{3} X-\left(9 a_{3}^{2}+a_{1}^{3} a_{3}\right),
$$

with non-vanishing 1 -form $\eta^{\prime}=d X /\left(2 Y+a_{1} X+3 a_{3}\right)$. There is an isogeny $\phi: C \rightarrow C^{\prime}$ of degree 3 defined by

$$
\phi:(x, y) \mapsto(X, Y)=\left(x-\frac{a_{3} y}{x^{2}}+\frac{a_{3} x}{y}, y-\frac{a_{3}^{2} y}{x^{3}}-\frac{a_{3} x^{3}}{y^{2}}\right),
$$

and under this map $\phi^{*} \eta^{\prime}=\eta$. The kernel of this isogeny is precisely the subgroup $A$ of order 3 generated by $P_{0} \in C$ 
Proof. It is straightforward to check that the function $\phi$ defines a rational map of curves $C \rightarrow C^{\prime}$ with $\phi^{*} \eta^{\prime}=\eta$, for example using a computer algebra package. One calculates that the discriminant of the curve $C^{\prime}$ is $\Delta^{\prime}=a_{3}\left(a_{1}^{3}-27 a_{3}\right)^{3}$, and hence $C^{\prime}$ is a non-singular elliptic curve in Weierstrass form. Thus one concludes that $\phi$ is a non-singular map between smooth elliptic curves. Since the coordinate functions $X(x, y)$ and $Y(x, y)$ have poles only on the subgroup $A$ of $E$ we see that $A$ is precisely the kernel of the map.

Remark 5.4. The curve $C^{\prime}$ was obtained by the following procedure. First, consider the map $\sigma: C \rightarrow C$ defined using the group structure on $E$ by $\sigma(P)=P+P_{0}$ where $P_{0}=(0,0)$ is a generator of $A$. One computes that $\sigma(x, y)=\left(-a_{3} y / x^{2},-a_{3}^{2} y / x^{3}\right)$. Then $X=x+\sigma^{*} x+\left(\sigma^{*}\right)^{2} x$ and $Y=y+\sigma^{*} y+\left(\sigma^{*}\right)^{2} y$ must be Weierstrass coordinates for the quotient variety $E^{\prime}$ and hence satisfy a Weierstrass polynomial which one can solve for explicitly; this polynomial is the equation for $C^{\prime}$.

Proof of (5.2). We compute the effect of the maps $f^{*}$ and $q^{*}$ on modular forms, using the fact that the isogeny $C \rightarrow C^{\prime}$ of (5.3) exhibits the universal example of an isogeny of degree 3. Thus, the map $f^{*}: \mathrm{MF} \rightarrow \operatorname{MF}\left(\Gamma_{0}(3)\right)$ sends $c_{i} \in \mathrm{MF}_{k}(i=4,6)$ to

$$
\left(f^{*} c_{i}\right)\left(\phi: C \rightarrow C^{\prime}, \eta\right)=c_{i}(C, \eta),
$$

which can be read off from formulas found in [Sil86, p. 46] which express the $c_{i}$ s as polynomials $C_{i}\left(a_{1}, a_{2}, a_{3}, a_{4}, a_{6}\right)$ in the Weierstrass parameters $a_{1}, a_{2}, a_{3}, a_{4}, a_{6}$; in this case, we have $f^{*} c_{i}=C_{i}\left(a_{1}, 0, a_{3}, 0,0\right)$. The map $q^{*}: \operatorname{MF} \rightarrow \operatorname{MF}\left(\Gamma_{0}(3)\right)$ is described by

$$
\left(q^{*} c_{i}\right)\left(\phi: C \rightarrow C^{\prime}, \eta\right)=c_{i}\left(C^{\prime}, \eta^{\prime}\right)
$$

so that $q^{*} c_{i}=C_{i}\left(a_{1}, 0,3 a_{3},-6 a_{1} a_{3},-\left(9 a_{3}^{2}+a_{1}^{3} a_{3}\right)\right)$.

The effect of $h^{*}$ is computed using the 3-power isogeny [3]:C $\rightarrow C$. This isogeny acts on invariant 1 -forms by $[3]^{*} \eta=3 \eta$, so that

$$
\left(h^{*} c_{i}\right)([3]: C \rightarrow C, \eta)=c_{i}\left(C, \frac{1}{3} \eta\right)=3^{i} c_{i}(C, \eta) .
$$

The formula for $t^{*}$ is obtained from the others, using the identities (5.1), and the fact that $\operatorname{MF}\left(\Gamma_{0}(3)\right)$ is an integral domain. Thus, we have

$$
t^{*}\left(10 a_{1}^{4}\right)=t^{*}\left(9 f^{*}\left(c_{4}\right)+q^{*}\left(c_{4}\right)\right)=9 q^{*}\left(c_{4}\right)+f^{*}\left(h^{*}\left(c_{4}\right)\right)=90 a_{1}^{4},
$$

from which we see that $t^{*}\left(a_{1}^{2}\right)=\epsilon 3 a_{1}^{2}$ where $\epsilon \in\{ \pm 1\}$. We also have

$$
t^{*}\left(240 a_{1} a_{3}\right)=t^{*}\left(q^{*}\left(c_{4}\right)-f^{*}\left(c_{4}\right)\right)=f^{*}\left(h^{*}\left(c_{4}\right)\right)-q^{*}\left(c_{4}\right)=80 a_{1}^{4}-2160 a_{1} a_{3},
$$


whence $t^{*}\left(a_{1} a_{3}\right)=\frac{1}{3} a_{1}^{4}-9 a_{1} a_{3}$. The identity $t^{*}\left(\left(a_{1} a_{3}\right)^{2}\right)=t^{*}\left(a_{1}^{2}\right) t^{*}\left(a_{3}^{2}\right)$ gives $t^{*}\left(a_{3}^{2}\right)=$ $\epsilon\left(-\frac{1}{27} a_{1}^{6}+2 a_{1}^{3} a_{3}-27 a_{3}^{2}\right)$. Finally, we can use the identity $t^{*} f^{*}\left(c_{6}\right)=q^{*}\left(c_{6}\right)$ to show that $\epsilon=+1$.

\section{Some homotopy Classes Detected by $Q(3)$}

In this section we describe how the image of $J$ classes are detected in $Q(3)$; this will give a proof of (6.2) which we needed for the computation of $\pi_{*} \operatorname{TMF}\left(\Gamma_{0}(3)\right)$.

Recall that TMF admits an $E_{\infty}$ String orientation [AHR] which refines the Witten genus. We use this to detect elements in the image of $J$ in the homotopy of $Q(3)$, using the standard formalism which we review below.

Let $\gamma: X \rightarrow B$ String be the map classifying a stable string bundle over a pointed space $X$. Write $T(\gamma)$ for the Thom spectrum of $\gamma$, and define $\bar{T}(\gamma)$ and $\alpha$ by the cofiber sequence

$$
\Sigma^{-1} \bar{T}(\gamma) \stackrel{\alpha}{\rightarrow} S^{0} \rightarrow T(\gamma) \rightarrow \bar{T}(\gamma)
$$

Let $R^{\bullet}$ denote a cosimplicial $E_{\infty}$-ring spectrum, such that $R^{0}$ admits a string orientation, and let $Q=\operatorname{Tot} R^{\bullet}$. Then the composite $\Sigma^{-1} \bar{T}(\gamma) \stackrel{\alpha}{\rightarrow} S^{0} \rightarrow Q$ is detected by an element $e(\alpha) \in\left[\bar{T}(\gamma), R^{1}\right]$, defined by a diagram

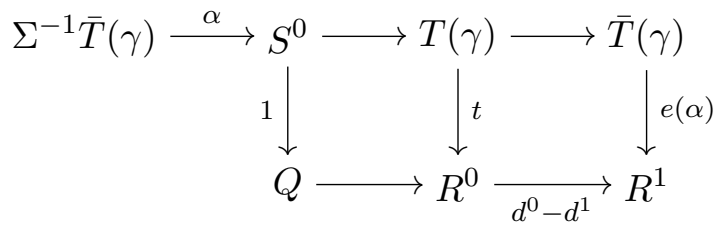

where $t$ is the map determined by the string orientation of $R^{0}$.

If $X=\Sigma Y$, then $\bar{T}(\gamma)=\Sigma^{\infty} \Sigma Y$ and $\alpha=J \tilde{\gamma}: \Sigma^{\infty} Y \rightarrow S^{0}$, where $\tilde{\gamma}: Y \rightarrow$ String is the adjoint of $\gamma$.

Proposition 6.1. Let $\gamma_{2 n}: S^{4 n} \rightarrow B$ String be the standard generator $(n \geq 2)$, so that $\alpha_{2 n}=J \tilde{\gamma}_{2 n}: S^{4 n-1} \rightarrow S^{0}$ is the generator of the image of J. Then $e\left(\alpha_{2 n}\right): S^{4 n} \rightarrow$ $R^{1} \rightarrow R_{\mathbb{Q}}^{1}$ is given by

$$
u_{n} \cdot\left(\left(d^{0}\right)_{*}\left(b_{2 n}\right)-\left(d^{1}\right)_{*}\left(b_{2 n}\right)\right) \in \pi_{4 n} R^{1} \otimes \mathbb{Q},
$$

where

$$
Q(x)=\frac{x}{\exp _{F}(x)}=\exp \left[2 \sum_{k \geq 1} b_{2 k} \frac{x^{2 k}}{2 k !}\right] \in H^{*}\left(\mathbb{C P}^{\infty}, \pi_{*} R^{0} \otimes \mathbb{Q}\right)
$$


is the Hirzebruch series associated to the given string orientation of $R^{0}$, and $u_{n}=1$ if $n$ is even and $u_{n}=2$ if $n$ is odd.

Proof. This is "standard"; a proof appears, for instance, in [AHR].

In particular, taking $R^{\bullet}$ to be the cosimplicial ring associated to the building complex of $\S 5$, and $Q=Q(3)=\operatorname{Tot}\left(R^{\bullet}\right)$, we see that $e\left(\alpha_{2 n}\right) \in \pi_{4 n} R^{1} \approx$ $\pi_{4 n} \operatorname{TMF}\left(\Gamma_{0}(3)\right) \times \pi_{4 n} \mathrm{TMF}$ is a class which modulo torsion has the name

$$
\left(u_{n} \cdot\left(q^{*} G_{2 n}-f^{*} G_{2 n}\right), u_{n} \cdot\left(3^{2 n}-1\right) G_{2 n}\right),
$$

where $G_{2 n} \in \mathrm{mf}_{2 n} \otimes \mathbb{Q}$ is the unnormalized Eisenstein series with $q$-expansion

$$
G_{2 n}(q)=-\frac{B_{2 n}}{4 n}+\sum_{m \geq 0} q^{m} \sum_{d \mid m} d^{2 n-1} .
$$

Corollary 6.2. There is an element in $\pi_{8} \operatorname{TMF}\left(\Gamma_{0}(3)\right)$ which maps to $a_{1} a_{3} \in$ $\pi_{8} \operatorname{TMF}\left(\Gamma_{1}(3)\right)$.

Proof. The element is given by $e\left(\gamma_{4}\right): S^{8} \rightarrow \operatorname{TMF}\left(\Gamma_{0}(3)\right)$, since $240 G_{4}=c_{4}$, so that $q^{*} G_{4}-f^{*} G_{4}=\frac{1}{240}\left[\left(a_{1}^{4}+24 a_{1} a_{3}\right)-\left(a_{1}^{4}-216 a_{1} a_{3}\right)\right]=a_{1} a_{3}$.

A number of elements in $\pi_{*} S^{0}$ are detected in $\pi_{*} Q(3)$; we hope to provide calculations of these in a future paper.

\section{Connective Models for $\operatorname{TMF}\left(\Gamma_{0}(3)\right)$}

By a "connective model" of a $v_{2}$-periodic ring spectrum $R$, we mean a connective spectrum $X$ such that $L_{K(2)} X \approx L_{K(2)} R$. More optimistically, we can ask that $L_{K(1) \vee K(2)} X \approx R_{p}^{\wedge}$. Even more optimistically, we may hope that $X$ is a ring spectrum, or even an $E_{\infty}$-ring.

Thus, the periodic spectrum of topological modular forms TMF comes with a canonical connective model tmf, which is itself an $E_{\infty}$-ring. There seems to be no known construction of a connective $\operatorname{tmf}\left(\Gamma_{0}(3)\right)$ which is also an $E_{\infty}$-ring.

In this section, all results are at the prime 2. Cohomology refers to mod 2 cohomology. We will make much use of the fact that $H^{*} \operatorname{tmf} \approx A \otimes_{A(2)} \mathbb{Z} / 2$.

Our first result is to construct a simple finite complex and a map of this complex into $\operatorname{TMF}\left(\Gamma_{0}(3)\right)$ such that the extension over TMF is a weak equivalence. We 
will think of this as a recognition complex. Then if we can map this complex into other naturally occurring module spectra we can determine if such a spectrum is a connective model for $\operatorname{TMF}\left(\Gamma_{0}(3)\right)$.

We begin with a finite complex $X=\mathrm{bo}_{1}$, which is the 7-skeleton of bo; that is, $X=S^{0} \cup_{\nu} e^{4} \cup_{\eta} e^{6} \cup_{2 \iota} e^{7}$. Let $Y=\Sigma^{7} D \mathrm{bo}_{1}$; that is, $Y$ is the Spanier-Whitehead dual of $\mathrm{bo}_{1}$, shifted so that the bottom cell is in dimension 0 .

Theorem 7.1. There is a map $f: \Sigma^{6} X \rightarrow Y$ so that the composite $S^{6} \rightarrow \Sigma^{6} X \rightarrow Y$ is $\nu^{2}$ on the bottom cell.

Proof. The proof is straightforward after computing $\pi_{*} Y$ through dimension 14 . The following is the Adams $E_{2}$-term in the usual way of presenting such charts.

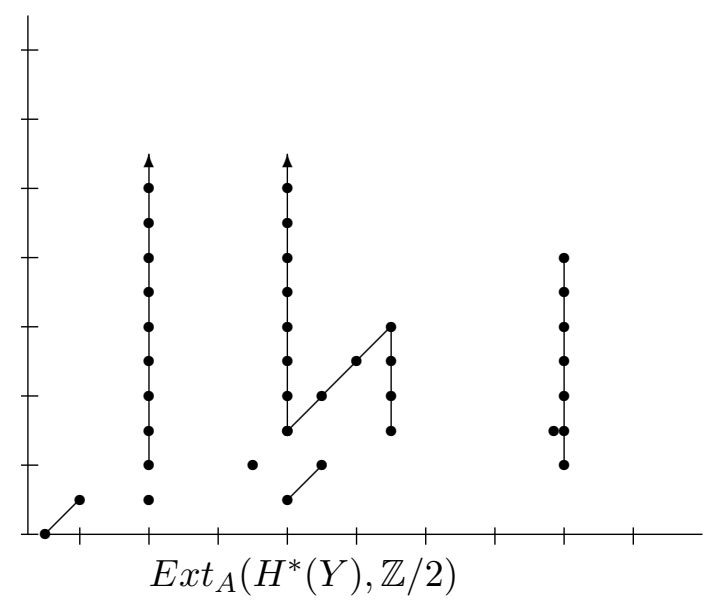

From this chart it is easy to see that the class which begins the map $\Sigma^{6} X \rightarrow Y$ sends the bottom class to a class in the six stem. Clearly, $\nu$ on this class is zero and this allows an extension over the four skeleton of $X$. The last Moore space maps in dimensions 11 and 12, both of which are zero.

The following result is our recognition principle. Let $Z=Y \cup_{f} C \Sigma^{6} X$.

Proposition 7.2. There is a map $g: \Sigma^{17} Z \rightarrow \operatorname{TMF}\left(\Gamma_{0}(3)\right)$ whose extension to $\Sigma^{17} Z \wedge$ $\mathrm{TMF} \rightarrow \operatorname{TMF}\left(\Gamma_{0}(3)\right)$ is a weak equivalence. 
Proof. This follows from the calculations of $\pi_{*} \operatorname{TMF}\left(\Gamma_{0}(3)\right)$ we made in $\S 4$. In particular, $g$ is constructed so that on the bottom cell it is given by $x: S^{17} \rightarrow$ $\operatorname{TMF}\left(\Gamma_{0}(3)\right)$.

We will use this result to show that various naturally occurring spectra $X$ are copies of covers of $\operatorname{TMF}\left(\Gamma_{0}(3)\right)$. In each case, a complete homotopy calculation of $X$ looks like a connective version of $\operatorname{TMF}\left(\Gamma_{0}(3)\right)$; this allows the construction of a map from $Z$ to $X$ just as in the proof of (7.2). When $X$ is a tmf-module, the map extends to a map $Z \wedge$ tmf to $X$.

The first example is the bit in MString which begins in dimension 16. Recall that the cohomology of $M$ String is free over $A \otimes_{A(2)} \mathbb{Z} / 2$. In dimension 16 there is a free generator and starting in dimension 20 there begins an extended $A(2)$-module $A(2) /\left(\mathrm{Sq}^{1}, \mathrm{Sq}^{5}, \mathrm{Sq}^{6}\right)$. It was determined in [DM82] that these two pieces are connected by $2 \nu$. The homotopy of the resulting tmf module was computed in [GM95]. An inspection of that calculation shows that this module is just a connected version of $\pi_{*} \operatorname{TMF}\left(\Gamma_{0}(3)\right)$.

We write $\operatorname{tmf}\left(\Gamma_{0}(3)\right)$ for this connected model of $\operatorname{TMF}\left(\Gamma_{0}\right)$. It is of special interest, since it has a good chance of being a ring spectrum. The homotopy of $\pi_{*} \operatorname{tmf}\left(\Gamma_{0}(3)\right)$ is described in [GM95]; in terms of the spectral sequence calculation of $\pi_{*} \operatorname{TMF}\left(\Gamma_{0}(3)\right)$ given in $\S 4$, the homotopy of $\operatorname{tmf}\left(\Gamma_{0}(3)\right)$ corresponds to the part of the $E_{2}^{s, t}$-term for which (i) $s \leq t-s$ (i.e., below the line of slope one), and (ii) whose $E_{1}$-names only include non-negative powers of the element $\Delta$.

Remark 7.3. The module $\mathbb{Z} / 2 \oplus \Sigma^{4} A(2) /\left(\mathrm{Sq}^{1}, \mathrm{Sq}^{5}, \mathrm{Sq}^{6}\right)$ admits the following nice description: it is isomorphic as a module to $H^{*}\left(\mathrm{bo}_{1}\right)^{\otimes 2} / \Sigma_{2}$, the symmetric coinvariants of $H^{*} \mathrm{bo}_{1}$.

In [GM95] it is also shown that there is an extended $A(2)$-module $M$ beginning in dimension 44 which is

$$
A(2)\left\{a_{44}, a_{49}\right\} /\left(\mathrm{Sq}^{1} a_{44}, \mathrm{Sq}^{5} a_{44}, \mathrm{Sq}^{7} a_{44}+\mathrm{Sq}^{2} a_{49}, \mathrm{Sq}^{4} a_{49}+\mathrm{Sq}^{6} \mathrm{Sq}^{3} a_{44}\right) .
$$

The Adams spectral sequence for this extended $A(2)$-module is easy to compute. Let $M_{1}=A(2)\left\{a_{5}\right\} / A(2)\left\{\mathrm{Sq}^{2} a_{5}, \mathrm{Sq}^{4} a_{5}\right\}$, and $M_{2}=A(2)\left\{a_{0}\right\} / A(2)\left\{\mathrm{Sq}^{1} a_{0}, \mathrm{Sq}^{5} a_{0}, \mathrm{Sq}^{13} a_{0}\right\}$. Then we have a short exact sequence

$$
M_{2} \rightarrow M \rightarrow M_{1}
$$


The Ext chart for $M_{1}$ is computed in [DM82]. The module $M_{2}$ fits into an exact sequence

$$
M_{2} \rightarrow A(2) /\left(\mathrm{Sq}^{1}, \mathrm{Sq}^{5}\right) \rightarrow A(2)\left\{a_{13}\right\} /\left(\mathrm{Sq}^{1}, \mathrm{Sq}^{2}, \mathrm{Sq}^{10}\right) .
$$

The Ext-chart for the right module is computed in [DM82] while the middle module is just $H^{*}$ bsp. Thus all parts are easily computed and there is a non-trivial connecting homomorphism in the sequence to compute $\operatorname{Ext}_{A(2)}(M)$. Again there are no possible Adams differentials. Its homotopy agrees with the 11 homotopy skeleton of the fiber of a map from $\operatorname{tmf}\left(\Gamma_{0}(3)\right) \rightarrow$ bo $\vee \Sigma^{8}$ bo. The first map picks up the unit and the second picks up $a_{1} a_{3}$.

These two results suggest that a possible path toward understanding MString is via these module maps and others like them. We have the orientation now thanks to Ando, Hopkins, and Rezk.

The $A(2)$-module structure of $A \otimes_{A(2)} \mathbb{Z} / 2$ is known. It is most easily described in terms of bo-Brown-Gitler spectra. Compare [GJM86]. The first two after the unit are $\Sigma^{8} \mathrm{bo}_{1}=\Sigma^{8} X$ and $\Sigma^{16} \mathrm{bo}_{2}$. Because of a differential in the Adams spectral sequence, these two pieces are connected. We have the following:

Proposition 7.4. $K(2)$-locally, there is a cofiber sequence

$$
\Sigma^{32} \mathrm{TMF} \rightarrow\left(\Sigma^{8} \mathrm{bo}_{1} \cup \Sigma^{16} \mathrm{bo}_{2}\right) \wedge \mathrm{TMF} \rightarrow \mathrm{TMF}\left(\Gamma_{0}(3)\right) .
$$

The proof consists of computing the homotopy of the middle spectrum and then applying the "recognition principle".

\section{Some Calculations in the Building COMPlex}

We want to understand how the map $\delta=q^{*}-f^{*}: \mathrm{MF}_{*} \rightarrow \mathrm{MF}_{*}\left(\Gamma_{0}(3)\right)$ works, which in turn gives us a good understanding of the corresponding map $\operatorname{TMF} \rightarrow \operatorname{TMF}\left(\Gamma_{0}(3)\right)$ in homotopy.

Proposition 8.1. We have

$$
\delta\left(\Delta^{2^{r}(2 k+1)}\right)=a_{1}^{3 \cdot 2^{r+1}} a_{3}^{2^{r+1}(4 k+1)}+\text { higher terms in } a_{1} \quad(\bmod 2) .
$$

Proof. To get the formula, recall that

$$
f^{*}(\Delta)=-27 a_{3}^{4}+a_{1}^{3} a_{3}^{3}, \quad q^{*}(\Delta)=-19683 a_{3}^{4}+2187 a_{1}^{3} a_{3}^{3}-81 a_{1}^{6} a_{3}^{2}+a_{1}^{9} a_{3} .
$$


This gives

$$
f^{*}(\Delta) \equiv\left(a_{3}^{4}+a_{1}^{3} a_{3}^{3}\right), \quad q^{*}(\Delta) \equiv a_{3}^{4}+a_{1}^{3} a_{3}^{3}+a_{1}^{6} a_{3}^{2}+a_{1}^{9} a_{3} \quad(\bmod 2) .
$$

Formally setting $a_{1}=t$ and $a_{3}=1$, the answer is obtained by determining the first non-zero term in

$$
\left(1+t^{3}+t^{6}+t^{9}\right)^{2^{r}(2 k+1)}-\left(1+t^{3}\right)^{2^{r}(2 k+1)} \quad(\bmod 2) .
$$

Since this expression is equal to $\left(1+t^{3}\right)^{2^{r}(2 k+1)}\left(\left(1+t^{6}\right)^{2^{r}(2 k+1)}-1\right)$, it is not hard to see that the leading term is $t^{3 \cdot 2^{r+1}}$.

Proposition 8.2. We have

$$
\delta\left(c_{4}^{k}\right)=2^{4+\nu_{2}(k)}\left[(\text { odd }) a_{1}^{4(k-1)} a_{1} a_{3}+\text { higher terms in } a_{1}\right]
$$

and

$$
\delta\left(c_{4}^{k} c_{6}\right)=2^{3}\left[(\text { odd }) a_{1}^{4 k+2} a_{1} a_{3}+\text { higher terms in } a_{1}\right] .
$$

Proof. The first equality is a straightforward application of the following lemma to the identity $q^{*}\left(c_{4}\right)=f^{*}\left(c_{4}\right)+2^{4}\left(15 a_{1} a_{3}\right)$. The second equality can be derived from the first together with the identity $q^{*}\left(c_{6}\right)=f^{*}\left(c_{6}\right)+2^{3}\left(63 a_{1}^{2} a_{1} a_{3}+756 a_{3}^{3}\right)$.

Lemma 8.3. If $d>1$, then

$$
\left(u+2^{d} v\right)^{k}=u^{k}+2^{d+\nu_{2}(k)} g(u, v),
$$

where $g(u, v) \in \mathbb{Z}[u, v]$ has the form $g(u, v)=($ odd $) u^{k-1} v+$ higher terms in $v$.

\section{REFERENCES}

[AHR] Matthew Ando, Michael J. Hopkins, and Charles Rezk, The string orientation of tmf, in preparation.

[AHS01] M. Ando, M. J. Hopkins, and N. P. Strickland, Elliptic spectra, the Witten genus and the theorem of the cube, Invent. Math. 146 (2001), no. 3, 595-687. MR 1869850

[Beh] Mark Behrens, Buildings, elliptic curves, and the $K(2)$-local sphere, arxiv:math.AT/0510026.

[Beh06] A modular description of the K(2)-local sphere at the prime 3, Topology 45 (2006), no. 2, 343-402. MR MR2193339 (2006i:55016)

[Del75] P. Deligne, Courbes elliptiques: formulaire d'après J. Tate, Modular functions of one variable, IV (Proc. Internat. Summer School, Univ. Antwerp, Antwerp, 1972), Springer, Berlin, 1975, pp. 53-73. Lecture Notes in Math., Vol. 476. MR MR0387292 (52 \#8135) 
[DM82] Donald M. Davis and Mark Mahowald, Ext over the subalgebra $A_{2}$ of the Steenrod algebra for stunted projective spaces, Current trends in algebraic topology, Part 1 (London, Ont., 1981), CMS Conf. Proc., vol. 2, Amer. Math. Soc., Providence, RI, 1982, pp. 297-342. MR MR686123 (85a:55018)

[GJM86] Paul G. Goerss, John D. S. Jones, and Mark E. Mahowald, Some generalized Brown-Gitler spectra, Trans. Amer. Math. Soc. 294 (1986), no. 1, 113-132. MR MR819938 (87d:55006)

[GM95] Vassily Gorbounov and Mark Mahwolald, Some homotopy of the cobordism spectrum $\mathrm{MO}\langle 8\rangle$, Homotopy theory and its applications (Cocoyoc, 1993), Contemp. Math., vol. 188, Amer. Math. Soc., Providence, RI, 1995, pp. 105-119. MR MR1349133 (96i:55010)

[HBJ94] Friedrich Hirzebruch, Thomas Berger, and Rainer Jung, Manifolds and modular forms, Vieweg, 1994, Translated by Peter S. Landweber.

[HK01] Po Hu and Igor Kriz, Real-oriented homotopy theory and an analogue of the Adams-Novikov spectral sequence, Topology 40 (2001), no. 2, 317-399. MR MR1808224 (2002b:55032)

[HM] M. J. Hopkins and M. Mahowald, From elliptic curves to homotopy theory, 1998 preprint, at http://hopf.math.purdue.edu.

[KM85] Nicholas M. Katz and Barry Mazur, Arithmetic moduli of elliptic curves, Annals of Mathematics Studies, vol. 108, Princeton University Press, Princeton, NJ, 1985. MR MR772569 (86i:11024)

[KW] Nitu Kitchloo and W. Stephen Wilson, On fibration related to real spectra, to appear.

[Sil86] J. H. Silverman, The arithmetic of elliptic curves, Graduate Texts in Mathematics, Springer-Verlag, 1986.

Mark Mahowald

Department of Mathematics

Northwestern University

2033 Sheridan Rd.

Evanston, IL 60208

E-mail: mark@math.northwestern.edu

Charles Rezk

Department of Mathematics

University of Illinois at Urbana-Champaign

1409 W. Green St.

Urbana, IL 61801

E-mail:rezk@math.uiuc.edu 